\title{
Expressed sequence tags (ESTs) from immune tissues of turbot (Scophthalmus maximus) challenged with pathogens
} Belén G Pardo1, Carlos Fernández ${ }^{1}$, Adrián Millán'1, Carmen Bouza1, Araceli Vázquez-López ${ }^{1}$, Manuel Vera1 ${ }^{1}$, José A Alvarez-Dios ${ }^{2}$, Manuel Calaza², Antonio Gómez-Tato ${ }^{3}$, María Vázquez ${ }^{4}$, Santiago Cabaleiro ${ }^{4}$, Beatriz Magariños ${ }^{5}$, Manuel L Lemos ${ }^{5}$, José M Leiro ${ }^{5}$ and Paulino Martínez*1

\footnotetext{
Address: ${ }^{1}$ Departamento de Genética. Facultad de Veterinaria, Universidad de Santiago de Compostela, Campus de Lugo, 27002 Lugo, Spain, ${ }^{2}$ Departamento de Matemática Aplicada, Facultad de Matemáticas, Universidad de Santiago de Compostela, 15782 Santiago de Compostela Spain, ${ }^{3}$ Departamento de Geometría y Topología, Facultad de Matemáticas, Universidad de Santiago de Compostela, 15782 Santiago de Compostela, Spain, ${ }^{4}$ Cluster de la Acuicultura de Galicia (CETGA), Punta de Couso s/n, 15965, Aguiño (Ribeira), A Coruña, Spain and ${ }^{5}$ Departamento de Microbiología y Parasitología. Universidad de Santiago de Compostela, 15782 Santiago de Compostela, Spain

Email: Belén G Pardo - mariabelen.gomez@usc.es; Carlos Fernández - carlos.fernandez.lopez@usc.es; Adrián Millán - adrian.millan@usc.es; Carmen Bouza - mcarmen.bouza@usc.es; Araceli Vázquez-López - aravazq@usc.es; Manuel Vera - manuel.vera@usc.es; José A AlvarezDios - joseantonio.alvarez.dios@usc.es ; Manuel Calaza - mcalaza@usc.es; Antonio Gómez-Tato - antonio.gomez.tato@usc.es; María Vázquez - ruizocenda@cetga.org; Santiago Cabaleiro - cabaleiro@cetag.org; Beatriz Magariños - beatriz.magarinos@usc.es; Manuel L Lemos - manuel.lemos@usc.es; José M Leiro - josemanuel.leiro@usc.es; Paulino Martínez* - paulino.martinez@usc.es

* Corresponding author
}

Published: 25 September 2008

BMC Veterinary Research 2008, 4:37 doi:10.1/86/1746-6/48-4-37
Received: 23 June 2008

Accepted: 25 September 2008

This article is available from: http://www.biomedcentral.com/I746-6/48/4/37

(c) 2008 Pardo et al; licensee BioMed Central Ltd.

This is an Open Access article distributed under the terms of the Creative Commons Attribution License (http://creativecommons.org/licenses/by/2.0), which permits unrestricted use, distribution, and reproduction in any medium, provided the original work is properly cited.

\begin{abstract}
Background: The turbot (Scophthalmus maximus; Scophthalmidae; Pleuronectiformes) is a flatfish species of great relevance for marine aquaculture in Europe. In contrast to other cultured flatfish, very few genomic resources are available in this species. Aeromonas salmonicida and Philasterides dicentrarchi are two pathogens that affect turbot culture causing serious economic losses to the turbot industry. Little is known about the molecular mechanisms for disease resistance and hostpathogen interactions in this species. In this work, thousands of ESTs for functional genomic studies and potential markers linked to ESTs for mapping (microsatellites and single nucleotide polymorphisms (SNPs)) are provided. This information enabled us to obtain a preliminary view of regulated genes in response to these pathogens and it constitutes the basis for subsequent and more accurate microarray analysis.
\end{abstract}

Results: A total of 12584 cDNAs partially sequenced from three different cDNA libraries of turbot (Scophthalmus maximus) infected with Aeromonas salmonicida, Philasterides dicentrarchi and from healthy fish were analyzed. Three immune-relevant tissues (liver, spleen and head kidney) were sampled at several time points in the infection process for library construction. The sequences were processed into 9256 high-quality sequences, which constituted the source for the turbot EST database. Clustering and assembly of these sequences, revealed 3482 different putative transcripts, 1073 contigs and 2409 singletons. BLAST searches with public databases detected significant similarity (e-value $\leq$ le-5) in $1766(50.7 \%)$ sequences and 816 of them $(23.4 \%)$ could be functionally annotated. Two hundred three of these genes (24.9\%), encoding for defence/immunerelated proteins, were mostly identified for the first time in turbot. Some ESTs showed significant 
differences in the number of transcripts when comparing the three libraries, suggesting regulation in response to these pathogens. A total of 191 microsatellites, with 104 having sufficient flanking sequences for primer design, and I I 58 putative SNPs were identified from these EST resources in turbot.

Conclusion: A collection of 9256 high-quality ESTs was generated representing 3482 unique turbot sequences. A large proportion of defence/immune-related genes were identified, many of them regulated in response to specific pathogens. Putative microsatellites and SNPs were identified. These genome resources constitute the basis to develop a microarray for functional genomics studies and marker validation for genetic linkage and QTL analysis in turbot.

\section{Background}

The turbot (Scophthalmus maximus; Scophthalmidae; Pleuronectiformes) is a commercially valuable flatfish that has been intensively cultured for the last decade. Its production has steadily increased to 7120 tonnes in 2006 (80\% from Spain; FEAP, 2006) and represents one of the most promising aquaculture species in Europe. However, disease outbreaks in turbot have occurred frequently and losses due to infections constitute a serious problem for its culture $[1,2]$. The use of antibiotics, vaccines and fish health management practices has partially solved the problem, but the achievement of large-scale production in the highly competitive world market requires enhancing resistance of cultured fish to diseases. Information on the immune response of turbot is still limited, and little is known about host-pathogen interactions in fish species. The screening and identification of immune-relevant genes is essential to analyze the genetic basis for infection, immunity and resistance to pathogens of economic relevance in aquaculture. Expressed sequence tag (EST) analysis is a powerful approach to provide a rapid and efficient method to go from expressed sequences to genes. ESTs are essential for studies of gene function $[3,4]$, but are also useful to identify polymorphic gene markers, such as microsatellites and single nucleotide polymorphisms (SNPs) [5-8]. These markers are the basis for genetic and physical mapping, and for comparative genome analysis [9-11]. From a practical perspective, maps can be applied for assisted selection programmes (MAS) and eventually for identification of genes related with quantitative traits (QTL) [12,13]. In addition, ESTs constitute the basic resources to develop microarrays for functional genomics studies [14].

EST sequence resources are rapidly growing in molecular databases. However, the number of ESTs in fish is generally scarce, excluding some model species and Atlantic salmon among cultured fish [15-19]. The scarcity of EST resources in cultured fish limits the use of modern functional genomic approaches for selective breeding purposes [20]. Among flatfish, aquaculture production has been successfully achieved in turbot, Japanese flounder and Atlantic halibut. Compared to the very large efforts for the development of EST resources in Japanese flounder (8856 ESTs) and Atlantic halibut (17659 ESTs) [21-28], EST resources in turbot are scarce (3171 ESTs). Less than 800 sequences have been deposited to date in the NCBI nucleotide database, most of them from anonymous microsatellite searching [29].

With the aim of increasing the genomic resources in turbot and identifying relevant genes for immunity, three cDNA libraries were constructed from mRNA isolated from immunity-related tissues of turbot (liver, spleen and head kidney) at different times after infection with Aeromonas salmonicida and the scuticociliate parasite Philasterides dicentrarchi. These pathogens are responsible for important disease outbreaks in turbot, as well as in other culture fish species [30-33]. Our main goal was to obtain the most accurate information possible to address functional genomic studies on disease resistance. However, the use of non-normalized cDNA libraries made it feasible to get a preliminary picture of the turbot genetic response to pathogens through analyzing transcript distribution among infected $v s$. control libraries. A total of 12584 ESTs were sequenced and compared to GenBank database and a large array of defence or immune-related genes was identified. Also, this large scale EST study increased the number of putative markers for mapping. A total of 191 microsatellites, of which 104 exhibited sufficient flanking sequences for primer design, and 2197 good quality SNPs were identified for the first time in turbot. The cDNA sequences generated will serve as a basis for microarray construction. This first EST study in turbot will provide the support for further research into the genetics, genomics and even proteomics of this important aquaculture species.

\section{Results and Discussion cDNA libraries and ESTs}

EST analysis is an efficient and fast method for gene discovery $[15,17]$. In Pleuronectiformes, this approach has been recently applied in Japanese flounder (Paralichthys olivaceus; [21,22,24,25]), winter flounder (Pseudopleuronectes americanus; [34]), flounder (Platichthys flesus; $[35,36]$ ) and Atlantic halibut (Hippoglossus hippoglossus; 
[26-28]. However, this order comprises around 600 species, many of them of great economic value both for fisheries and farming. In this study, we have addressed the construction of an EST database for the identification of genes related with immunity and defence in turbot.

The three cDNA libraries constructed held at least $2.5 \times$ $10^{6}$ primary recombinant clones. The directional cloning approach used for construction of cDNA libraries ensured that CDNA inserts appeared mostly in the same orientation within the vector. Clones were sequenced from the 3' end with the vector primer T7 to obtain large gene-specific genomic regions for future oligo-microarray design. The libraries used were non-normalized and, as usually observed [16], substantial redundancy was obtained (around 74\%). This approach allowed an analysis of the turbot response to specific pathogens by comparing the amount of transcripts across all genes or groups of genes classified in functional categories. A total of 12584 ESTs were sequenced. After trimming and vector removal 9256 high quality ESTs were obtained (Table 1), showing an average length of $409 \mathrm{bp}$. Their sequences are available in the dbEST NCBI database under numbers FE943103FE952358.

EST projects generate a large number of redundant sequences due to the random selection of cDNAs from tissue libraries, especially when libraries are non-normalized and a high number of clones are sequenced. Clustering redundant sequences is a critical step to identify genes. The program CAP3 http://seq.cs.iastate.edu./ was used to cluster EST sequences using the default parameters. As shown in Table 1, clustering yielded 3482 unique turbot sequences: 2409 singletons (69.2\%) and 1073 contigs (30.8\%) comprising 6847 ESTs (6.4 sequences/contig) and an average length of $527 \mathrm{bp}$. Figure $1 \mathrm{~A}$ shows the histogram distribution of contig sizes. Although most contigs showed two (46.7\%) or three-tofive $(30.3 \%)$ sequences, a small number of highly

Table I: Summary statistics of ESTs from turbot libraries

\begin{tabular}{lcc}
\hline & Number & $\%$ \\
\hline Good-quality ESTs & 9256 & \\
Redundant sequences & 6847 & 74.0 \\
Unique sequences & 3482 & 36.0 \\
$\quad$ Contigs & 1073 & 30.8 \\
$\quad$ Singletons & 2409 & 69.2 \\
Unique sequences with no BLAST hits & 1716 & 49.3 \\
Unique sequences with BLAST hits & 1766 & 50.7 \\
$\quad$ BLASTN & 1091 & 61.8 \\
BLASTX & 675 & 38.2 \\
Unique sequences with functional annotation & 816 & 23.4 \\
$\quad$ Contigs & 489 & 59.9 \\
Singletons & 327 & 40.1
\end{tabular}

expressed genes were also detected. As shown in Figure $1 \mathrm{~B}$, the beta-globin contig (407 ESTs) and five others contained more than 100 ESTs, which represents a high proportion $(1212 / 9256=13.1 \%)$ over the total redundancy $(74.0 \%)$. Most of these ESTs shared homology with genes involved in transport, protein metabolism and response to stress, all of which related to defence. At the other extreme, $77.0 \%$ of the 1073 contigs were $\leq 5$ times redundant, indicating that most of these unique sequences represent rare mRNAs and that these libraries provide a rich source of sequence information.

\section{Gene annotation}

ESTs were identified by BLAST searches against nucleotide database at GenBank. Due to the low representation of fish genes, a protein-based homology strategy was also used in the international database searches. Protein sequences have been demonstrated to be more suitable to detect homology over long periods of evolutionary time [37]. Our EST database pprovided a graphical view of all contigs, their PROSITE/PRINTS protein patterns and a search interface by keywords, microsatellite, gene, and UniGene/GO/KEGG information. Tools to search for sequences and markers based on annotations, to perform local BLAST searches, and to select sequences for a prospective microarray were also included. These tools used RepeatMasker http://www.repeatmasker.org/ for masking low-complexity sequences and OligoArray $2.1 \mathrm{http} / / /$ berry.engin.umich.edu/oligoarray2_1/ to predict secondary structures and potential cross-hybridization.

As shown in Table 1, 1716 (49.3\%) unique sequences displayed no significant similarity to known sequences or ESTs in the public databases, whereas the remaining 1766 $(50.7 \%)$ showed significant matches with e-values $\leq 1 \mathrm{e}-5$. Among these, BLAST database searches allowed assignment of putative function to 816 sequences $(23.4 \%)$. In spite of their lower frequency among unique sequences, contigs were annotated more frequently $(59.9 \%)$ than singletons $(40.1 \%)$. As in other ESTs fish studies, the lower percentage of annotated singletons suggests that these are either novel fish-specific or rapidly evolving genes [38]. Also, it is possible that bioinformatic errors could have a greater impact on singletons, since they are unique sequences whose information cannot be contrasted with other sequences in the database. The lower annotation success regarding similar genomic projects $[28,39]$ was probably related to the read length (around $500 \mathrm{bp}$ ) and specially the direction (from the 3'end) of sequencing. The 3' untranslated region (UTR) is approximately double the length of the 5'UTR according to GenBank fish entries. So, what is gained in specificity for microarray oligo-design is lost for gene annotation. 
A

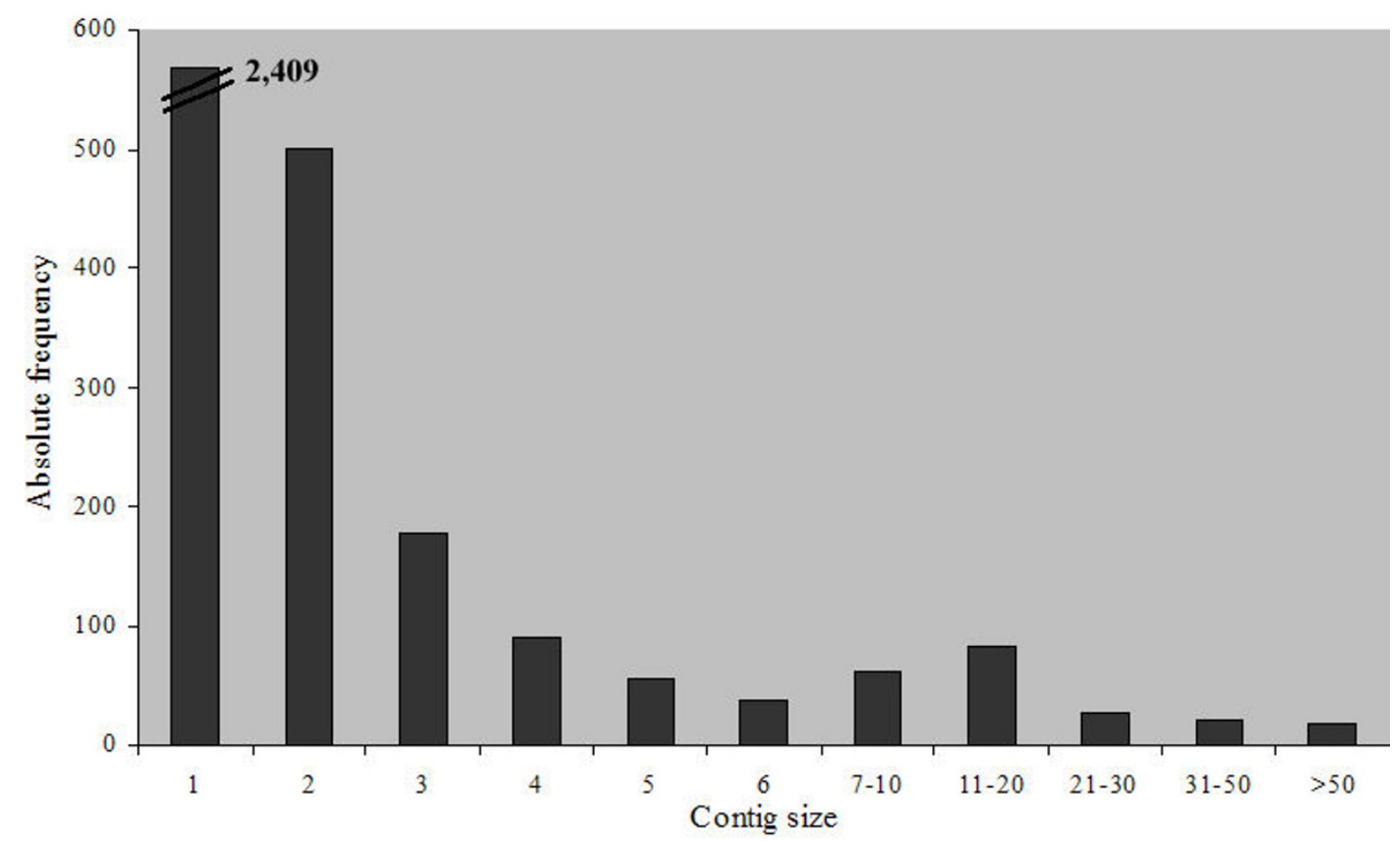

B

\begin{tabular}{cccccc}
\hline Gene & Contig size & e-value & $\%$ Hom & Biological process & BLAST \\
\hline Hepcidin precursor & 70 & $3 \mathrm{e}-21$ & 83 & - & BLASTN \\
Lysozyme C precursor & 76 & $8 \mathrm{e}-42$ & 100 & Response to stress & BLASTX \\
Trypsinogen 1 precursor & 86 & $7 \mathrm{e}-21$ & 67 & Proteolysis & BLASTX \\
Serotransferrin & 88 & $5 \mathrm{e}-11$ & 85 & Ion transport & BLASTN \\
Trypsinogen 1 & 114 & $4 \mathrm{e}-88$ & 69 & Proteolysis & BLASTX \\
cDNA clone D. labrax & 148 & $8 \mathrm{e}-22$ & 82 & - & BLASTN \\
Ribosomal protein S27a & 155 & $3 \mathrm{e}-32$ & 68 & Prot. metabol. process & BLASTX \\
Ribosomal protein S2 & 171 & 0.0 & 93 & Biosyntethic process & BLASTN \\
Hemoglobin alpha-1 chain & 219 & $6 \mathrm{e}-32$ & 86 & Transport & BLASTX \\
Beta type globin & 407 & $3 \mathrm{e}-127$ & 81 & Transport & BLASTN \\
\hline
\end{tabular}

\section{Figure I}

Sequence prevalence distribution of the identified contigs from turbot libraries. (A) Absolute frequency histogram showing contig size (number of sequences) distribution. (B) Functional and BLAST hits confidence characteristics of the ten largest contigs. Only biological function according to GO terms has been included. 
All unique sequences were annotated based on similarity using BLASTX or BLASTN [40] in the public databases GenBank NR and Unigene. The multiple annotations provided greater assurance about gene description and frequency of annotation than in a single database. The use of consensus sequences allowed sequences without significant similarity regions with a known protein (e.g., 5' or 3' noncoding regions) to be annotated if they were members of an annotated contig. All hits with e-value $\leq 1 \mathrm{e}-5$ and their associated alignments were stored in the database and tracked with any associated functional annotation. We also ran AutoFACT [41] on all sequences in the database. It is interesting to note that while AutoFACT was able to come up with more function-specific information than our online tool (provided external database hits were found), it was not able to annotate as many sequences as our online custom tool.

Annotated ESTs (816) were classified into functional categories according to GO terms [42] (77.0\%), 367 among contigs $(75.1 \%)$ and 261 among singletons $(79.8 \%)$. A single sequence very often showed several GO terms in the same ontology, so we try to group them using a single more general category. Overly specific categories were also collapsed into more general terms. Any terms related to defence/immunity were always retained given their interest in this study. Biological process, molecular function and cellular component categories are shown in Figures 2, 3 and 4, respectively. According to these criteria, biological processes associated with proteins (synthesis, metabolism and proteolysis) were the largest annotated categories, though a significant group related to transport and response to stress did appear. In accordance with this, a large proportion of sequences were classified into structural constituent of ribosome and protein-related functions (binding, peptidase activity) regarding molecular function. A remarkably high proportion of annotated sequences were categorized as oxidoreductase activity. Finally, most gene activity was located into the ribosome, followed by the membrane, nucleus and the extracellular and cytoplasm constituents of the cell.

\section{Immune genes}

Systematic classification of annotated sequences using available bioinformatics tools (GO, KEGG) provides a useful information to analyze the functional profile of annotated genes. In our study, we were especially interested in identifying immune genes or in a broader sense those genes regulated in response to specific pathogens of turbot. So, we analyzed the relationship of our annotated

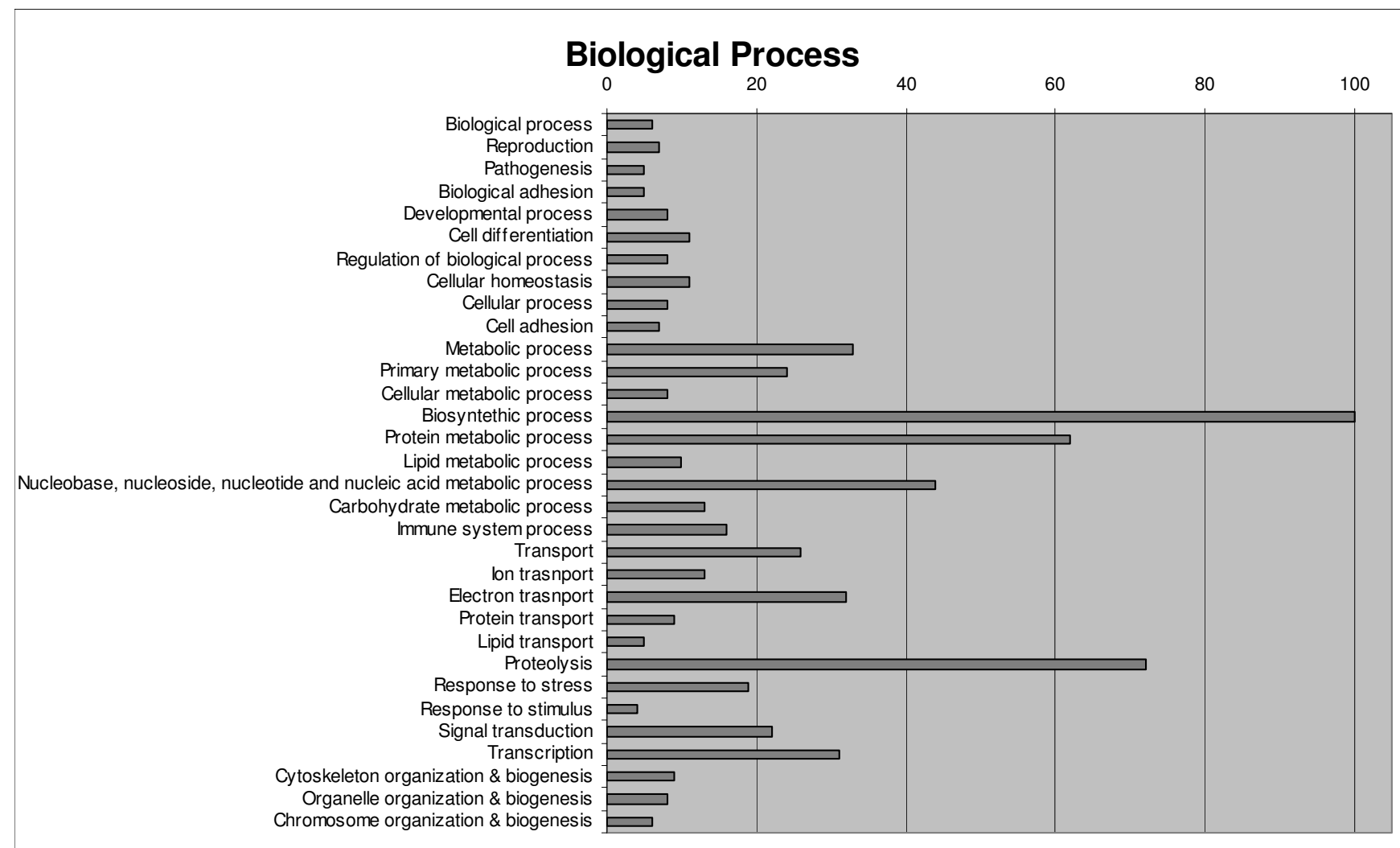

Figure 2

Classification of turbot unique sequences in biological processes categories following Gene Ontology (GO). 


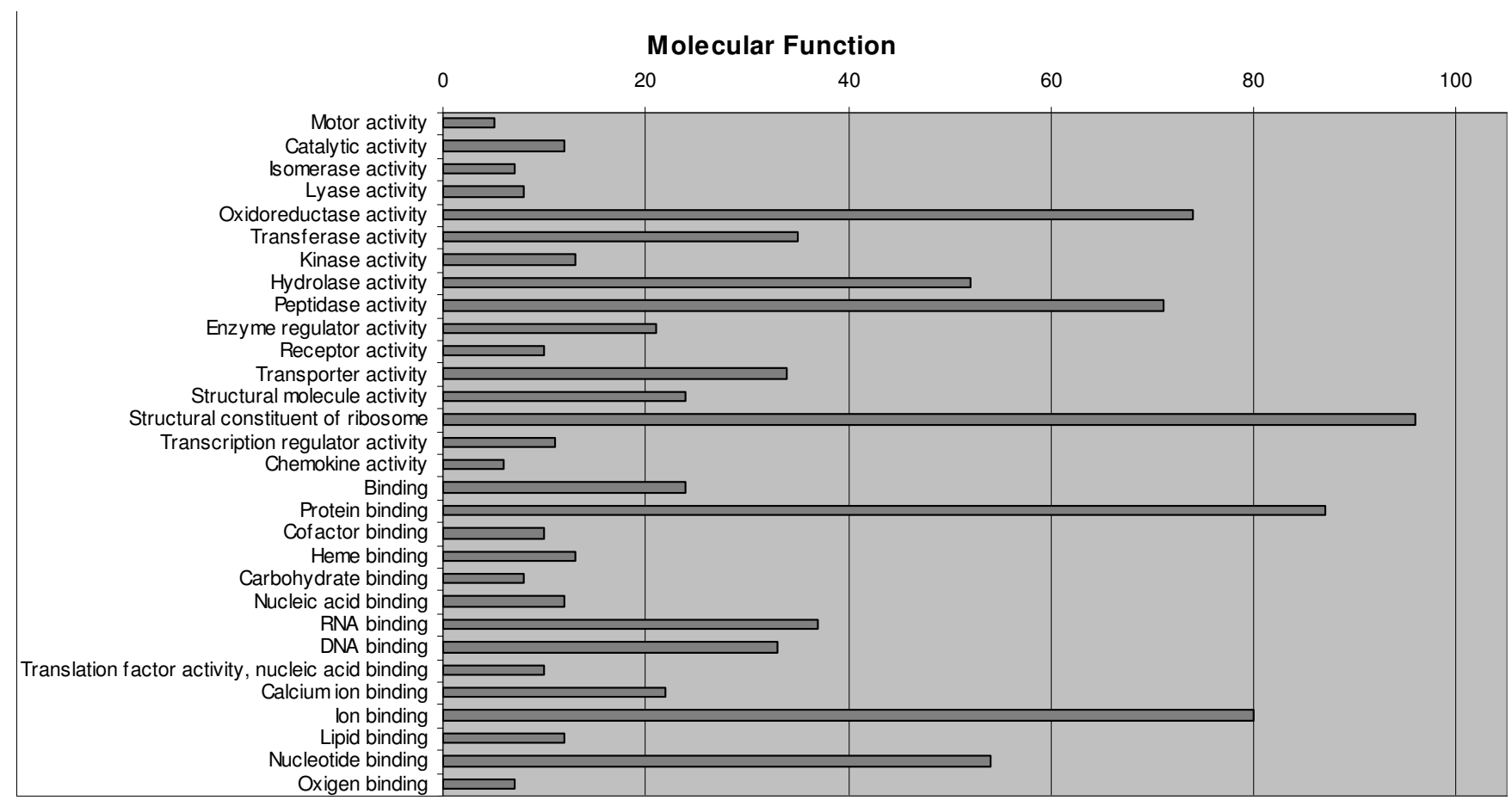

Figure 3

Classification of turbot unique sequences in molecular function categories following Gene Ontology (GO).

genes with defence or immunity using scientific literature information available on vertebrates. According to this analysis, 203 genes out of 816 annotated (24.9\%) appeared related to defence or immunity in our work (Table 2). This observation is in agreement with the important role of liver, spleen and head kidney in the immune response of fish.

Like in mammals, the immune system of fish is composed of non-specific and specific defence. The innate immune response is an important and highly developed defence mechanism against pathogens in fish [43]. Examples of innate immunity include anatomic barriers, mechanical removal of pathogens, bacterial antagonism, pattern-recognition receptors, antigen-nonspecific defence compounds, complement pathways, phagocytosis, and inflammation [43]. In Table 2, a list of defence/immunerelated genes that were found three or more times in our libraries is shown. Complement related genes were predominant $(7.9 \%)$ followed by apoptosis $(4.9 \%)$ and immunoglobulin (Ig)-related (3.9\%) genes. Glutathione S-transferase, heat shock proteins and cytochrome P450, elastases, major histocompatibility complex (MHC) and coagulation factors involved in innate immunity were also present, as well as others like interferon, perforin, hepcidin, nephrosin or alpha-2-macroglobulin. Interestingly, the majority of our immune-related cDNAs $(75.0 \%)$ were reported for the first time in turbot, even though some of them have important roles in the immune response like B-cell linker, chemotaxin, complement components, IgD, IgM, interferon stimulated gene 12 (b2), lipopolysaccharide (LPS)-binding protein, natural killer (NK)-lysine type 1, peptidoglycan recognition protein, skin mucus lectin and tumour necrosis factor (TNF) receptor associated factor2, among others. Previously, only a hepcidin [44] and a natural killer cell enhancing factor [45] had been characterized in turbot.

The availability of a large number of sequences from immune-related cDNA libraries, both from non-infected fish (controls) as well as from fish challenged with specific pathogens, suggested a comparison of transcript profiles to identify genes regulated in response to these pathogens. Four or more sequences per gene (contig) are the minimum necessary to get statistical power to check the null hypothesis of even distribution of sequences among the three libraries. Ninety six genes presented four or more sequences in our libraries. However, taking into account the large number of tests performed, we decided to use a more conservative set of genes (with 6 or more sequences) to avoid type I errors. This new set comprised 72 genes, whose distribution among libraries is shown in Additional file 1 together with their probability of departure from the null hypothesis using a chi-square test. To use all information available and to get higher statistical power, we decided to perform an additional analysis by compar- 


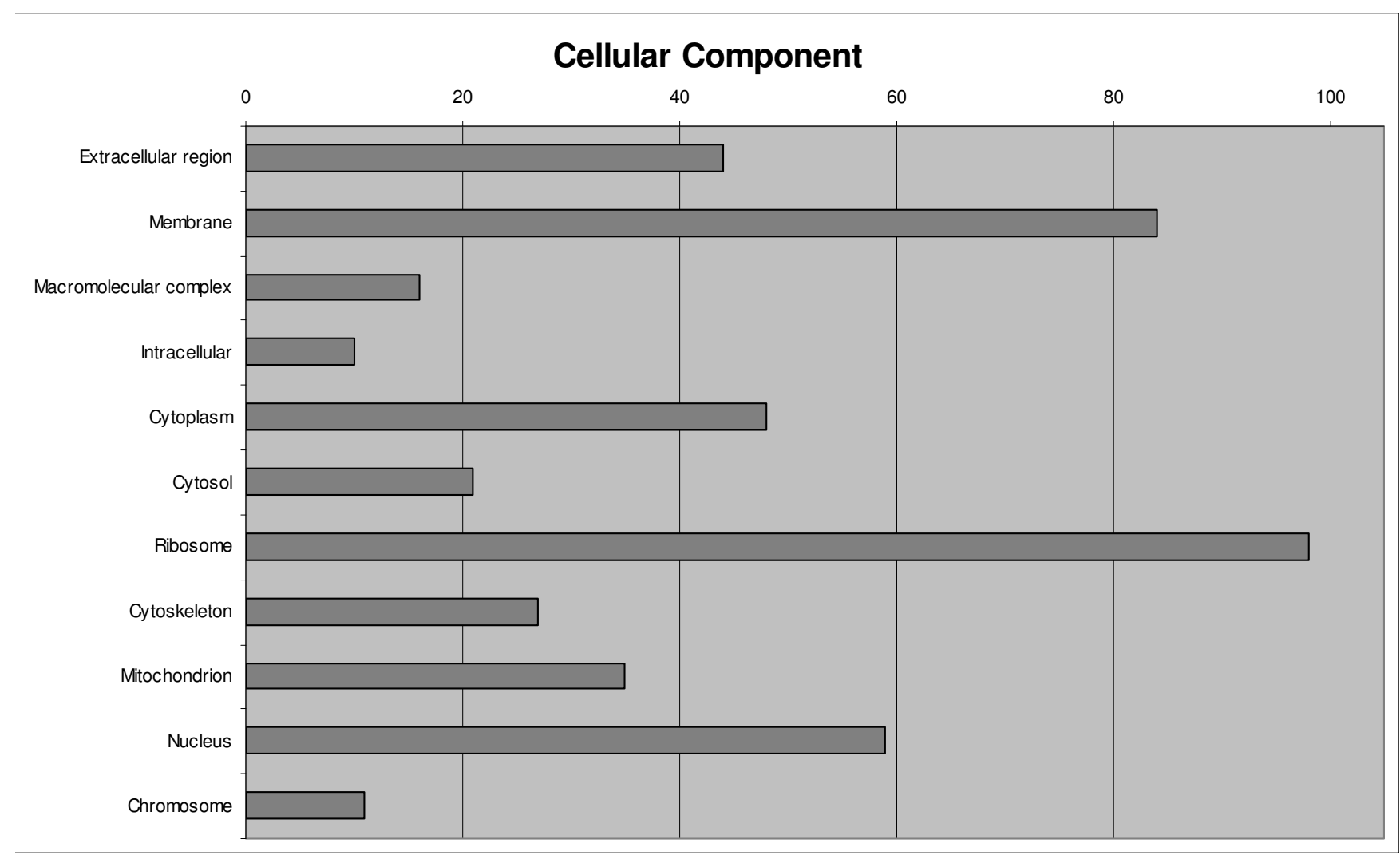

Figure 4

Classification of turbot unique sequences in cellular component categories following Gene Ontology (GO).

Table 2: Defence and immune-related annotated ESTs from turbot libraries

\begin{tabular}{lcc}
\hline Genes & No. unique sequences & $\%$ \\
\hline Complement related & 16 & 7.9 \\
Apoptosis related & 10 & 4.9 \\
Immunoglobulin related & 8 & 3.9 \\
Glutathione S-transferase & 7 & 3.5 \\
Elastase & 6 & 2.9 \\
Cytochrome P450 & 6 & 2.9 \\
Major histocompatibility complex & 5 & 2.5 \\
Coagulation factor & 5 & 2.5 \\
Interferon related & 3 & 1.5 \\
Perforin & 3 & 1.5 \\
Hepcidin precursor & 3 & 1.5 \\
Nephrosin & 3 & 1.5 \\
Alpha-2-macroglobulin & 3 & 1.5 \\
Other genes & 119 & 58.6 \\
\hline Total & & 24.9 \\
\hline
\end{tabular}

"No. unique sequences" refers to the total amount of the different annotated contigs and singletons for each gene class listed in the Table. "Total percentage" of defence/immune-related genes is referred to the number of unique annotated sequences from turbot libraries (816). ing the profiles of all genes grouped according to GO categories (see Additional file 2). Though this approach could be mixing genes with opposite expression patterns, it could provide significant trends associated to specific functions for complementing the "individual-level gene" analysis. Bonferroni correction for multiple tests was considered in this case, because of the higher statistical power achieved with this approach.

As expected, defence/immune-related genes appeared overrepresented in the set of 72 genes $(37.5 \%)$ with regard to the total number of annotated genes in our libraries $(24.9 \%)$. Some genes apparently responded in a similar way to both pathogens, being down- (myosin, nephrosin and several peptidases) or up-regulated (actin and lysozyme) regarding control. However, most genes responded to only one pathogen. The different infection profile of the pathogens used in this study, a bacterium (A. salmonicida) and a parasite ( $P$. dicentrarchi), is expected to stimulate a specific set of genes in the host. So, protein biosynthesis was up-regulated in response to Aeromonas salmonicida, as suggested the increased amount or ribosome-related and elongation factor proteins, and their correspondent GO terms (see Additional file 2). Also, a 
general down-regulation of genes involved in proteolytic activity in response to this bacterium (chymotrypsin B precursor, trypsinogen 1 , chymotrypsinogen 2 , trypsynogen-like serin protease and elastase precursor) was observed, in turn correlated to the lower presence of peptidase and proteolysis GO terms in this library. The possibility that $A$. salmonicida could be blocking these genes cannot be ruled out, since it would facilitate the infection process. Additionally, it was noteworthy that several iron metabolism-related genes turned out to be up-regulated after infection with A. salmonicida (haptoglobin fragment 1 , globin-related proteins, hepcidin precursor). The high relevance of iron for bacteria infection could explain this observation. The up-regulation of hepcidin after bacteria infection has been recently described in turbot and gilthead seabream $[44,46]$. Finally, some other immune relevant genes like MHC II alpha and beta antigens, complement C9, thrombin, chemotaxin, bactericidal permeability-increasing protein, apolipoprotein A-IV3, heat shock protein 90 beta and chemotaxin showed significant up-regulation in response to $A$. salmonicida.

However, the strongest genetic signature was observed in response to $P$. dicentrarchi infection. Specific genes were exclusively detected in this library and sometimes at high frequencies, like those homologous to Oryzias latipes and Paralichthys olivaceous cDNA clones and to mitochondrial ATP synthase alpha-subunit gene. Functional annotation of these unknown genes now appears relevant to understand the response of turbot to this pathogen. The same was reflected when GO categories were analyzed (see Additional file 2). A large number of differentially regulated gene categories $(P=0)$ appeared associated to $P$. dicentrarchi library according to the different GO criteria. Cytoskeleton organization and biogenesis, as well as carbohydrate metabolic process appeared up-regulated categories among biological processes. Transporter and hydrolase activities, as well as protein, ion and heme binding also were overexpressed regarding molecular function classification. Finally, cytoskeleton and cytosol appeared as the highest active cellular components. Looking at specific categories, a significant increase in the expression of antioxidant genes, like glutathione peroxidase and glutathione-S-transferase, was observed. These genes play a pivotal role to prevent cellular damage due to the increased reactive oxygen species (ROS) during infection [47]. Paramá et al. [48] have recently demonstrated the increase in intracellular ROS by proteases of turbot kidney in response to this pathogen. Tissue trauma or invasion by pathogens induces changes in the quantities of several macromolecules in animal body fluids, which comprise one aspect of the acute phase response (APR). In fish, APR proteins include pentraxins, serum amyloid P, several components of the complement system, transferrin and thrombin. Up-regulation of pentraxin and sero- transferrin was observed in response to $P$. dicentrarchi in our study. Transferrin up-regulation could be related to the increase in inflammation and enhanced oxidative stress typical of infections with this parasite [48]. Finally, up-regulation of other important components of the immune response such the profilin and lysozyme was observed. Profilin-like protein has been reported as a tolllike receptor (TLR) 11 ligand in some parasites [49]. TLRs are evolutionary conserved transmembrane proteins that recognize a unique pattern of molecules derived from pathogens or damaged cells, triggering robust but defined innate immune responses [49].

\section{Markers-containing ESTs}

EST studies can also provide resources for the identification of polymorphic DNA markers such as microsatellites and SNPs. In our study, screening of EST sequences for short tandem repeats (2-6 bp) identified 191 microsatellites using a conservative criterion ( $\geq 6$ and $\geq 8$ repeats for tri/tetra/penta and dinucleotide motifs, respectively). Of these, 120 had significant hits in BLAST with e-value cut off $\leq 1 e-5$ and 71 were annotated. Most microsatellites were dinucleotide (128) and trinucleotide (56), while only 6 tetranucleotide and 1 pentanucleotide were found. Among these 191 ESTs sequences, 104 contained sufficient flanking sequences length for primer design. Fifty of these 104 microsatellites-containing ESTs were contigs, therefore the in silico comparison of the sequences included in these contigs allowed us to identify 11 putative polymorphic microsatellites.

Because of their abundance within the genome, SNPs are the most common type of genetic markers [50] for studying complex genetic traits and genome evolution [51]. In turbot there have no been reports on SNP identification so far. The use of non-normalized libraries and a large number of individuals in library construction made possible the identification of 2197 good quality SNPs. After the three filters used in the QualitySNP pipeline [52], we finally detected 2197 real and 1158 true SNPs (Table 3), representing a rate of 1.39 and 0.74 SNPs per $100 \mathrm{bp}$, respectively. Real and true SNPs included 749 and 453 transitions, 974 and 558 transversions and 366 and 125 indels, respectively. With this pipeline, only clusters with at least 4 EST sequences were selected to minimize the detection of SNPs caused by sequencing errors. As shown in Table 4 the majority of SNPs were detected in contigs involving a larger number of sequences, which provides an additional proof of our SNP quality. The identification of microsatellite and SNPs markers within turbot ESTs will contribute to extend the turbot genetic map [10] after linkage analysis in reference families. Since these markers are linked to genes, they will be useful as Type I markers for population genomics scanning in this species and for comparative mapping and fish evolutionary studies. 
Table 3: Summary statistics of SNP identification from turbot EST resources

\begin{tabular}{lrr}
\hline & Real SNPs & \multicolumn{2}{l}{ True SNPs andSNPs } \\
\hline Total sequences analysed & & 12584 \\
Number of contigs & 257 & \\
Total SNPs detected & 2197 & 1158 \\
SNP frequency & $1.39 / 100 \mathrm{bp}$ & $0.74 / 100 \mathrm{bP}$ \\
Total number of transitions & 749 & 453 \\
C/T & 556 & 344 \\
A/G & 193 & 109 \\
Total number of transversions & 974 & 558 \\
A/T & 161 & 87 \\
A/C & 352 & 214 \\
T/G & 251 & 130 \\
C/G & 210 & 127 \\
Total number of indels & 366 & 125 \\
Tri-allelic polymorphisms & 99 & 21 \\
Tetra-allelic polymorphisms & 9 & $\mathrm{I}$ \\
\hline
\end{tabular}

Real SNPs are those which passed quality filters I and 2 using the pipeline QualitySNP and true SNPs are the highest quality SNPs passing the three filters (see Methods).

\section{Conclusion}

To our knowledge, this is the first report on a large transcriptional analysis in turbot providing new genomic resources in this important European aquaculture species. This study describes a collection of 9256 ESTs representing 3482 unique sequences obtained from three directionally cloned cDNA libraries from $S$. maximus, all of these being novel ESTs for this species. Therefore, this is a valuable EST collection, which increases genomic resources of turbot and enhances the genomic tools available for non-model fish species. The transcript profile comparison among the three libraries allowed the identification of putative genes generally or specifically related with infections in turbot. In addition, a high number of putative microsatellite and SNP EST-markers are now available for turbot map and highly useful for comparative mapping. These ESTs will be the basis for the develop-

Table 4: Real and true quality SNP distribution in contigs with 4 or more ESTs

\begin{tabular}{lrrr}
\hline & Number of contigs & Real SNPs & True SNPs \\
\hline with 4 sequences & 22 & 58 & 58 \\
with 5-10 sequences & 94 & 295 & 235 \\
with II-20 sequences & 75 & 437 & 266 \\
with 21-30 sequences & 28 & 322 & 215 \\
with 31-50 sequences & 21 & 289 & 110 \\
with > 50 sequences & 17 & 796 & 274 \\
& & & \\
Total & 257 & 2197 & 1158 \\
\hline
\end{tabular}

Real SNPs are those which passed quality filters $I$ and 2 using the pipeline QualitySNP and true SNPs are the highest quality SNPs passing the three filters (see Methods). ment of a turbot microarray, focused on the characterization of the transcriptional response to pathogen exposure.

\section{Methods \\ Tissue source and challenge}

Two batches of 40 individuals (20-30 g each) obtained from a mixture of heterogeneous genetic families were collected at a specialized turbot fish farm. Fish from each batch were challenged intraperitoneally with A. salmonic$i d a$ subsp. salmonicida and $P$. dicentrarchi, respectively $[53,54]$. The dose was adjusted to obtain around 50\% survival (LD50). The challenges were performed at the CETGA facilities in quarantine tanks. Fish were sacrificed prior to organ extraction using a lethal dose of MS222 anesthetic. In order to obtain mRNA representative of both innate and adaptive immune systems across the infection process, liver, spleen and head kidney tissues were collected from 5 sacrificed fish at five sampling points along the infective process for A. salmonicida (12 h, 1 day, 3 days, 7 days and 21 days post-inoculation) and at four sampling points for $P$. dicentrarchi ( 1 day, 3 days, 7 days and 15 days post-inoculation). Analogous batches of control fish were injected with saline serum and sampled at the same days of challenged fish. For each sample time, equal amounts of tissue from liver, kidney and spleen of each fish were pooled and immediately frozen in liquid nitrogen, constituting 15 pools for A. salmonicida (3 tissues $\times 5$ sampling points), 12 for $P$. dicentrarchi ( 3 tissues $\times 4$ sampling points) and the same pools for their respective controls. Each sample of pooled tissues was ground to a fine powder in a mortar and pestled with liquid nitrogen and stored at $-80^{\circ} \mathrm{C}$ until being used for RNA extraction. The use of pools of individuals at each sampling point was planned, both for identifying putative SNPs, as well as for averaging individual effects in future microarray analysis on turbot immune response.

\section{RNA isolation and cDNA library construction}

Total RNA was extracted from pooled tissues of control and infected fish using TRIZOL Reagent (Life Technologies) according to manufacturer's recommendations. RNA quality was assessed in a Bioanalyzer (Bonsai Technologies). RNA was quantified using gel and NanoDrop ${ }^{\circledR}$ ND1000 spectrophotometer (NanoDrop ${ }^{\circledR}$ Technologies Inc) estimations. Poly-A mRNA was isolated using the Dynabeads $^{\circledR}$ mRNA Purification Kit (INVITROGEN). Three cDNA libraries (from A. salmonicida and P. dicentrarchi infected fish and control) were directionally constructed (5' EcoRI, 3' XhoI), with equal amounts of RNA from each tissue at each sampling time, using the ZAP-cDNA Library Construction Kit (STRATAGENE) following manufacturer's instructions except for size fractioning. This was performed on cDNAs prior to ligation into vector and carried out with the SizeSep 400 Spun Columns (GE 
HEALTHCARE). To allow characterization of the inserts in a plasmid system the library was mass excised according to the manufacturer's recommendations, converting it from plaque forming units to a phagemid in E. coli SOLR strain. Manually isolated colonies were randomly picked and arrayed into 96-well microtiter plates containing liquid selective medium. Glycerol stocks of overnight cultures were prepared in 96 well plates and stored at $-80^{\circ} \mathrm{C}$.

\section{Sequencing and bioinformatics}

Plasmid DNA was isolated from around 4000 clones from each library using the DirectPrep ${ }^{\circledR} 96$ Miniprep kit (QIAGEN) and the Plasmid Miniprep96 kit (MILLIPORE) with a robotic platform (BIOMEK 3000), and checked by electrophoresis in 1\% agarose gels. One part of the purified DNA was sequenced following the ABI Prism BigDye ${ }^{\mathrm{TM}}$ Terminator v3.1 Cycle Sequencing Kit protocol on an ABI 3100 DNA sequencer (Applied Biosystems) and the other with the DTCS kit protocol on a CEQ2000 DNA sequencer (BECKMAN COULTER). All clones were sequenced from their 3' ends using a standard T7 primer to obtain the highest specific sequences of genes for oligomicroarray design. Those clones which suffered a systematic drop on sequencing signal after poly-A tails were sequenced from 5 ' end.

A bioinformatic tool was developed in order to process all data. Basecalling from chromatogram traces was performed by using PHRED $[55,56]$. Vector, poly-A tails and low-quality regions were trimmed from EST sequences using a custom Perl script, a local BLAST search engine and the trimmest utility from the EMBOSS suite. High quality ESTs (at least $100 \mathrm{bp}$ and PHRED score $\geq 20$ after removal of vector sequence, adapter, and poly-A tail) of both normal and infected libraries were combined and assembled to form clusters using CAP3 http:// seq.cs.iastate.edu./[57] with the overlapping identity percentage and minimum overlapping length parameters set to $>85.0 \%$ and $50 \mathrm{bp}$, respectively, in order to obtain highly reliable contig sequences. These contigs were manually revised to detect possible errors all along the bioinformatics process. ESTs that did not form contigs (singletons) and the contigs resultant of assemblage of multiple sequences were referred to as unique sequences. Singletons and consensus sequences of each contig (unique sequences) were compared against public databases. Unique sequences were searched by both Blastn and Blastx, and the corresponding outputs subsequently parsed using BioPerl for fish-relevant hits and significant UniGene information. GO, KEGG and COG terms were extracted from AutoFACT output, which in turn feeds from Tblastx and RPS-blast output. All results were stored in a mySQL database to be consulted and searched using a custom-designed, friendly AJAX web interface. The evalue cut off was $\leq 1 e-5$. All annotations thus obtained were complemented with AutoFACT output, whenever available.

\section{Bioinformatic mining of microsatellites and SNPs}

The set of 3482 unique sequences was searched for microsatellites using the program SPUTNIK http://espressosoft ware.com/pages/sputnik.jsp. The minimum repeat number used for this search was 8 for dinucleotide and 6 for tri-, tetra-and pentanucleotide microsatellites. Microsatellite-containing ESTs were identified as candidates for marker development if they presented enough flanking sequences on either side of the repeats for primer design. Detection of single nucleotide polymorphisms (SNPs) was done using the pipeline QualitySNP with default settings [52]. This program uses three filters for the identification of reliable SNPs: filter 1 screens for all potential SNPs; filter 2 uses a haplotype-based strategy to detect reliable SNPs; and filter 3 screens SNPs by calculating a confidence score based on sequence redundancy and quality. SNPs that pass filters 1 and 2 are called real SNPs, and those that pass all of the three filters are called true SNPs.

\section{Authors' contributions}

This work represents a collaboration between different institutions (Xunta de Galicia -local Government- and Universities) and turbot industry (CETGA- Centro Tecnológico Gallego de Acuicultura-). PM and CB identified the need for this investigation, designed and supervised the project. BGP and AV constructed the cDNA libraries. BGP, CF, and MVE performed all sequencing work, and revised and compiled this information for the subsequent bioinformatics analysis. Database for editing, clustering and functional annotation of genes was developed by JAA, $\mathrm{MC}$, and $\mathrm{AG}$ in close connection with PM, BGP, CB, CF and AM. These last two authors performed all bioinformatics analysis using this database. BM and MLL, for Aeromonas salmonicida, and JL, for Philasterides dicentrarchi, supplied virulent strains and performed all challenges at CETGA facilities. Maintenance, sacrifice, organ and RNA extraction from fish was done by MV and SC. The paper was written by BGP and mostly revised by PM and JAA. All authors read the manuscript and gave their approval.

\section{Additional material}

\footnotetext{
Additional file 1

Comparison of gene expression profiles regulated in response to turbot pathogens. The total number and distribution among libraries (Aeromonas, Philasterides, control) of sequences from contigs with 6 or more sequences is presented. The homology with public databases (e-value), the function and the probability of departure from the null hypothesis of even distribution of sequences among libraries is shown for each contig. Click here for file

[http://www.biomedcentral.com/content/supplementary/17466148-4-37-S1.doc]
} 


\section{Additional file 2}

Comparison of ESTs grouped according to GO terms among the three turbot cDNA libraries. contigs of annotated genes are shown grouped according to GO term categories (Molecular function; Biological process; Cellular component) and split into the three turbot libraries from which sequences were obtained. The expression pattern of each gene at each library is evaluated as the amount of sequences found. This pattern is compared among libraries and examined for its deviation from an even distribution among libraries by a chi-square test.

Click here for file

[http://www.biomedcentral.com/content/supplementary/17466148-4-37-S2.doc]

\section{Acknowledgements}

This study was supported by a Consellería de Pesca e Asuntos Marítimos and the Dirección Xeral de I+D - Xunta de Galicia project (2004/CP480). Authors wish to thank to Lucía Insua, Sonia Gómez, Ana Teijido and Susana Sánchez for technical assistance.

\section{References}

I. Toranzo AE, Novoa B, Romalde JL, Núñez S, Devesa S, Mariño E, Silva R, Martínez E, Figueras A, Barja JL: Microflora associated with healthy and diseased turbot (Scophthalmus maximus) from three farms in northwest Spain. Aquaculture 1993, I | 4: |89-202.

2. Iglesias R, Paramá A, Álvarez MF, Leiro J, Ubeira FM, Sanmartín ML: Philasterides dicentrarchi (Ciliophora: Scuticociliatida) expresses surface immobilization antigens that probably induce protective immune responses in turbot. Parasitology 2003, I 26:125-134.

3. Adams MD, Kelley JM, Gocayne JD, Dubnick M, Polymeropoulos MH, Xiao H, Merril CR, Wu A, Olde B, Moreno RF: Complementary DNA sequencing: expressed sequence tags and human genome project. Science 199I, 252:165I-I656.

4. Marra MA, Hillier L, Waterston RH: Expressed sequence tagsESTablishing bridges between genomes. Trends Genet 1998, I 4:4-7.

5. Liu ZJ, Karsi A, Dunham RA: Development of polymorphic EST markers suitable for genetic linkage mapping of catfish. Mar Biotechnol 1999, I:437-447.

6. Liu ZJ, Li P, Kocabas A, Ju Z, Karsi A, Cao D, Patterson A: Microsatellite-containing genes from the channel catfish brain: evidence of trinucleotide repeat expansion in the coding region of nucleotide excision repair gene RAD23B. Biochem Biophys Res Commun 200I, 289:3 I7-324.

7. He C, Chen L, Simmons M, Li P, Kim S, Liu Z): Putative SNP discovery in interspecific hybrids of catfish by comparative EST analysis. Anim Genet 2003, 34:445-448.

8. Serapion J, Kucuktas H, Feng J, Liu Z: Bioinformatic mining of type I microsatellites from expressed sequence tags of channel catfish (Ictalurus punctatus). Mar Biotechnol 2004, 6:364-377.

9. Murakawa K, Matsubara K, Fukushima A, Yoshii J, Okubo K: Chromosomal assignments of 3Vdirected partial cDNA sequences representing novel genes expressed in granulocytoid cells. Genomics 1994, 23:379-389.

10. Bouza C, Hermida M, Pardo BG, Fernández C, Castro J, Fortes G, Sánchez L, Presa P, Pérez M, Sanjuán A, Comesaña S, Álvarez JA, Calaza M, Cal R, Piferrer F, Martínez P: A microsatellite genetic map of the turbot (Scophthalmus maximus). Genetics 2007, I 77:2457-2467.

II. Moen T, Hayes B, Baranski M, Berg PR, Kjøglum S, Koop BF, Davidson WS, Omholt SW, Lien S: A linkage map of the Atlantic salmon (Salmo salar) based on EST-derived SNP markers. BMC Genomics 2008, 9:223.

12. Poompuang S, Hallerman EM: Toward detection of quantitative trait loci and marker-assisted selection in fish. Reviews Fish Sci 1997, 5:253-277.

13. Liu ZJ, Cordes JF: DNA marker technologies and their applications in aquaculture genetics. Aquaculture 2004, 238: I-37.
14. Jørgensen SM, Afanasyev S, Krasnov A: Gene expression analyses in Atlantic salmon challenged with infectious salmon anemia virus reveal differences between individuals with early, intermediate and late mortality. BMC Genomics 2008, 9: I79.

15. Hirono I, Aoki T: Expressed sequence tags of medaka (Oryzias latipes) liver mRNA. Mol Mar Biol Biotechnol I997, 6:345-350.

16. Lo J, Lee S, Xu M, Liu F, Ruan H, Eun A, He Y, Ma W, Wang W, Wen $Z$, Peng J: I5,000 Unique zebrafish EST clusters and their future use in microarray for profiling gene expression patterns during embryogenesis. Genome Res 2003, 13:455-466.

17. Clark MS, Edwards YJ, Peterson D, Clifton SW, Thompson AJ, Sasaki M, Suzuki Y, Kikuchi K, Watabe S, Kawakami K, Sugano S, Elgar G, Johnson SL: Fugu ESTs: new resources for transcription analysis and genome annotation. Genome Res 2003, I 3( I 2):2747-2753.

18. Rise ML, von Schalburg KR, Brown GD, Mawer MA, Devlin RH, Kuipers N, Busby M, Beetz-Sargent M, Alberto R, Gibbs AR, Hunt P, Shukin R, Zeznik JA, Nelson C, Jones SR, Smailus DE, Jones SJ, Schein JE, Marra MA, Butterfield YS, Stott JM, Ng SH, Davidson WS, Koop BF: Development and application of a salmonid EST database and cDNA microarray: data mining and interspecific hybridization characteristics. Genome Res 2004, I 4:478-490.

19. Jaillon O, Aury JM, Brunet F, Petit JL, Stange-Thomann N, Mauceli E, Bouneau L, Fischer C, Ozouf-Costaz C, Bernot A, Nicaud S, Jaffe D, Fisher S, Lutfalla G, Dossat C, Segurens B, Dasilva C, Salanoub M, Levy M, Boudet N, Castellano S, Anthouard V, Jubin C, Castelli V, Katinka M, Vacherie B, Biemont C, Skalli Z, Cattolico L, Poulain J, De Berardinis V, Cruaud C, Duprat S, Brottier P, Coutanceau JP, Gouzy J, Parra G, Lardier G, Chapple C, McKernan KJ, McEwan P, Bosak S, Kellis M, Volff JN, Guigo R, Zody MC, Mesirov J, Lindblad-Toh K, Birren B, Nusbaum C, Kahn D, Robinson-Rechav M, Laudet V, Schachter V, Quetier F, Saurin W, Scarpelli C, Wincker P, Lander ES, Weissenbach J, Roest Crollius $\mathrm{H}$ : Genome duplication in the teleost fish Tetraodon nigroviridis reveals the early vertebrate protokaryotype. Nature 2004, 43 I:946-957.

20. Chen SL, Xu MY, Hu SN, Li L: Analysis of immune relevant genes expressed in red sea bream (Chrysophrys major) spleen. Aquaculture 2004, 240: I I5-130.

21. Inoue S, Nam BH, Hirono I, Aoki T: A survey of expressed genes in Japanese flounder (Paralichthys olivaceus) liver and spleen. Mol Mar Biol Biotechnol 1997, 6:376-380.

22. Nam BH, Yamamoto E, Hirono I, Aoki T: A survey of expressed genes in the leukocytes of Japanese flounder, Paralichthys olivaceus, infected with Hirame rhabdovirus. Dev Comp Immunol 2000, 24:13-24.

23. Aoki T, Hirono I, Kim M-G, Katagiri T, Tokuda Y, Toyohara H, Yamamota E: Identification of viral induced genes in Ig+ leucocytes of Japanese flounder Paralichthys olivaceus, by differential hybridisation with subtracted and un-subtracted cDNA probes. Fish Shellfish Immunol 2000, 1 0:623-630.

24. Arma NR, Hirono I, Aoki T: Genes expressed in Japanese flounder Paralichthys olivaceus spleen: analysis of genes involved in immune function. Fisheries Sci 2005, 7 I: | 304- | 323.

25. Aoki T, Hirono I: Immune relevant genes of Japanese flounder, Paralichthys olivaceus. Comp Biochem Phys D 2006, I: I I5-I2I.

26. Park KC, Osborne JA, Tsoi SCM, Brown LL, Johnson SC: Expressed sequence tags analysis of Atlantic halibut (Hippoglossus hippoglossus) liver, kidney and spleen tissues following vaccination against Vibrio anguillarun and Aeromonas salmonicida. Fish Shellfish Immunol 2005, I 8:393-4I 5.

27. Galloway TF, Bardal T, Kvam SN, Dahle SW, Nesse G, Randol M, Kjorsvik E, Andersen O: Somite formation and expression of MyoD, myogenin and myosin in Atlantic halibut (Hippoglossus hippoglossus L.) embryos incubated at different temperatures: transient asymmetric expression of MyoD. J Exp Biol 2006, 209:2432-244l.

28. Douglas SE, Knickle LC, Kimball J, Reith ME: Comprehensive EST analysis of Atlantic halibut (Hippoglossus hippoglossus), a commercially relevant aquaculture species. BMC Genomics 2007, 8: 144.

29. Pardo BG, Fernández $C$, Hermida $M$, Vázquez $A$, Pérez $M$, Presa $P$, Calaza M, Alvarez-Dios JA, Comesaña AS, Raposo-Guillán J, Bouza C, Martínez P: Development and characterization of 248 novel microsatellite markers in turbot (Scophthalmus maximus). Genome 2007, 50:329-332. 
30. Toranzo $A E$, Barja JL: First report of furunculosis in turbot reared in floating cages in North-West of Spain. Bull Eur Ass Fish Pathol 1992, I2:147-149.

3I. Björnsdóttir B, Gudmundsdóttir S, Bambir SH, Gudmundsdóttir BK: Experimental infection of turbot, Scophthalmus maximus (L.), by Aeromonas salmonicida subsp. achromogenes and evaluation of cross protection induced by a furunculosis vaccine. J Fish Dis 2005, 28:181-188.

32. Iglesias R, Paramá A, Alvarez MF, Leiro J, Fernandez J, Sanmartin ML: Philasterides dicentrarchi (Ciliophora, Scuticociliatida) as the causative agent of scuticociliatosis in farmed turbot Scophthalmus maximus in Galicia (NW Spain). Dis Aquat Organ 200I, 46:47-55.

33. Paramá A, Arranz JA, Alvarez MF, Sanmartin ML, Leiro J: Ultrastructure and phylogeny of Philasterides dicentrarchi (Ciliophora, Scuticociliatia) from farmed turbot in NW Spain. Parasitology 2006, 132:555-564.

34. Douglas SE, Gallant JW, Bullerwell CE, Wolff C, Munholland J, Reith ME: Winter flounder expressed sequence tags: establishment of an EST database and identification of novel fish genes. Mar Biotechnol 1999, I:458-464.

35. Williams TD, Gensberg K, Minchin SD, Chipman JK: A DNA expression array to detect toxic stress response in European flounder (Platichthys flesus). Aquat Toxicol 2003, 65: | 14I-I57.

36. Sheader DL, Gensberg K, Lyons BP, Chipman K: Isolation of differentially expressed genes from contaminant exposed European flounder by suppressive, subtractive hybridisation. Mar Environ Res 2004, 58:553-557.

37. Pearson WR: Identifying distantly related protein sequences. Comput Appl Biosci 1997, 13:325-332.

38. Paschall JE, Oleksiak MF, VanWye JD, Roach JL, Whitehead JA, Wyckoff GJ, Kolell KJ, Crawford DL: FunnyBase: a systems level functional annotation of Fundulus ESTs for the analysis of gene expression. BMC Genomics 2004, 5:96.

39. Quilang J, Wang S, Li P, Abernathy J, Peatman E, Wang Y, Wang L, Shi Y, Wallace R, Guo X, Liu Z: Generation and analysis of ESTs from the eastern oyster, Crassostrea virginica Gmelin and identification of microsatellite and SNP markers. BMC Genomics 2007, 8:157.

40. Altschul SF, Gish W, Miller W, Myers EW, Lipman DJ: Basic local alignment search tool. J Mol Biol 1990, 215:403-410.

4I. Koski LB, Gray MW, Lang BF, Burger G: AutoFACT: An Automatic Functional Annotation and Classification Tool. BMC Bioinformatics 6:151.

42. Gene Ontology: tool for the unification of biology. The Gene Ontology Consortium. Nature Genet 2000, 25:25-29.

43. Ellis AE: Innate host defence mechanisms of fish against viruses and bacteria. Dev Comp Immunol 200I 25:827-839.

44. Chen SL, Li W, Meng L, Sha ZX, Wang ZJ, Ren GC: Molecular cloning and expression analysis of a hepcidin antimicrobial peptide gene from turbot (Scophthalmus maximus). Fish Shellfish Immunol 2007, 22:172-181.

45. Chen Y, Zhang YX, Fan TJ, Meng L, Ren GC, Chen SL: Molecular identification and expression analysis of the natural killer cell enhancing factor (NKEF) gene from turbot (Scophthalmus maximus). Aquaculture 2006, 261: I I86-1 I 93.

46. Cuesta A, Meseguer J, Esteban MA: The antimicrobial peptide hepcidin exerts an important role in the innate immunity against bacteria in the bony fish gilthead seabream. Mol Immunol 2008, 45:2333-2342.

47. Valko M, Rhodes CJ, Moncol J, Izakovic M, Mazur M: Free radicals, metals and antioxidants in oxidative stress-induced cancer. Chem Biol Interact 2006, 160:1-40.

48. Paramá A, Castro R, Arranz JA, Sanmartín ML, Lamas J, Leiro J: Scuticociliate cysteine proteinases modulate turbot leucocyte functions. Fish Shellfish Immunol 2007, 23:945-956.

49. Ishii KJ, Uematsu S, Akira S: 'Toll' gates for future immunotherapy. Curr Pharm Des 2006, 12:4135-4142.

50. Picoult-Newberg L, Ideker TE, Pohl MG, Taylor SL, Donaldson MA, Nickerson DA, Boyce-Jacino M: Mining SNPs from EST databases. Genome Res 1999, 9:167-174.

51. Syvanen AC: Accessing genetic variation: genotyping single nucleotide polymorphisms. Nat Rev Genet 200I, 2:930-942.

52. Tang J, Vosman B, Voorrips RE, Linden CG van der, Leunissen JAM: QualitySNP: a pipeline for detecting single nucleotide poly- morphisms and insertions/deletions in EST data from diploid and polyploid species. BMC Bioinformatics 2006, 7:438.

53. Magariños B, Santos Y, Romalde JL, Barja JL, Toranzo AE: Pathogenic activities of live cells and extracellular products of the fish pathogen Pasteurella piscicida. J Gen Microbiol 1992, | 38:249|-2498.

54. Paramá A, Iglesias R, Álvarez MF, Leiro J, Aja C, Sanmartín ML: Philasterides dicentrarchi (Ciliophora, Scuticociliatida): experimental infection and possible routes of entry in farmed turbot (Scophthalmus maximus). Aquaculture 2003, 21 7:73-80.

55. Ewing B, Hillier L, Wendl MC, Green P: Base-calling of automated sequencer traces using phred. I. Accuracy assessment. Genome Res 1998, 8: 175-185.

56. Ewing B, Green P: Base-calling of automated sequencer traces using phred. II. Error probabilities. Genome Res 1998, 8: $186-194$.

57. Huang $X$, Madan A: CAP3: a DNA sequence assembly program. Genome Res 1999, 9:868-877.
Publish with Biomed Central and every scientist can read your work free of charge

"BioMed Central will be the most significant development for disseminating the results of biomedical research in our lifetime. "

Sir Paul Nurse, Cancer Research UK

Your research papers will be:

- available free of charge to the entire biomedical community

- peer reviewed and published immediately upon acceptance

- cited in PubMed and archived on PubMed Central

- yours - you keep the copyright 